\title{
The effect of the inactive mutation on longevity, sex, rhythm and resistance to p-Cresol in Drosophila melanogaster
}

\author{
K. M. C. O'DELL \\ Institute of Genetics, University of Glasgow, Church Street, Glasgow G11 5JS, Scotland, UK
}

\begin{abstract}
Flies carrying the inactive (iav) mutation exhibit low locomotor activity and poor mating success, both of which are associated with a deficiency in the putative neurotransmitter, octopamine. Several other aspects of the iav mutant phenotype are described here. Male and female iav mutants show a small reduction in longevity but it is not clear whether this is a consequence of the iav mutation or their inactive phenotype. Young iav males show extended attractiveness to older courting males, which supports the notion that the $i a v$ gene has a role in post-eclosional maturation. The eclosion rhythm of iav mutants is normal, discounting the possibility of a role for octopamine in the maintenance of circadian rhythm. Flies carrying the iav mutation are highly susceptible to the octopamine analogue $p$-Cresol. Other phenotypically inactive flies show wild type levels of $p$-Cresol resistance. This is attributed to the deficiency of octopamine in iav mutants because low octopamine levels may be unable to out-compete the toxic effect of $p$-Cresol. Some inferences on the possible mode of action of the iav gene product are discussed.
\end{abstract}

Keywords: behaviour, courtship, Drosophila, longevity, octopamine, rhythm.

\section{Introduction}

Genetic and molecular approaches to the study of Drosophila melanogaster courtship may ultimately reveal underlying biochemical and developmental constraints associated with behaviour. Several mutations have now been isolated that alter specific aspects of Drosophila courtship behaviour (see Hall, 1982) but little is known about their mode of action. The inactive (iav) mutation, which was originally isolated by Kaplan (1977), provides one route by which we may understand the function of the putative Drosophila neurotransmitter, octopamine, during courtship and other behaviour.

The iav mutation and its allele $i a v^{2}$ (formerly hypoactive- $B^{1}$, Homyk \& Sheppard, 1977), were both isolated in flies showing locomotor-deficient phenotypes. Adult iav mutants show reductions in both the amount and speed of movement, exhibit poor reactivity and have several other abnormal locomotor-related phenotypes (O'Dell \& Burnet, 1988).

The mating success of flies carrying the iav mutation is markedly reduced. Poor mating success of iav males is primarily the consequence of their inactive phenotype, which precludes the maintenance of close contact during courtship. Homozygous iav and $i a v^{2}$ females show reductions in mating propensity and extended courtship durations. However, given that iav females have normal cuticular hydrocarbon compositions and receive a high intensity of courtship from mature males, they can be considered as attractive but unreceptive (O'Dell et al., 1989). Such behaviour is consistent with the aberrant perception and/or processing of incoming courtship information.

High performance liquid chromatography (HPLC) on flies carrying the iav mutation reveals that they have only 15 per cent of the wild type octopamine level but normal titres of dopamine and noradrenaline (O'Dell et al., 1987). The precise role of octopamine in $D$. melanogaster is unclear, but it has been shown to have neurotransmitter, neuromodulator and neurohormone functions in several invertebrate species (David \& Coulon, 1985). As yet it is unclear whether this deficit in octopamine is the cause or the consequence of the iav mutant phenotype, but it is consistent with the prediction of a neural focus for the $i a v^{2}$ mutation by Homyk (1977).

It should be possible to use iav mutants to establish the role of octopamine in the Drosophila nervous system and its consequences for behaviour and 
development. Livingstone \& Tempel (1983) found a small octopamine deficit in rhythmless period mutants, and the effect of the iav mutation on circadian rhythm is decribed here. The effects of $p$-Cresol, a structural analogue of octopamine, on the survival of iav mutants is also described. $p$-Cresol is known to inhibit bovine dopamine $\beta$-hydroxylase (Goodhart et al., 1987), the Drosophila equivalent of which is a candidate for the iav gene product.

A further consequence of the iav mutation appears to be a small developmental delay in the onset of some adult locomotor and sexual behaviours (O'Dell \& Burnet, 1988; O'Dell et al., 1989). This apparent delay may affect other developmentally regulated posteclosional maturation events and the consequences of the iav mutation for the onset of male sexual behaviour and longevity are described.

\section{Materials and methods}

\section{Fly strains}

Unless otherwise stated, all comparisons are made between flies carrying the $i a v^{1}$ allele of Kaplan (1977) and wild type. As described previously (O'Dell \& Burnet, 1988), to avoid the effects of genetic background the $i a v^{1}$ allele was crossed in to two wild type strains, Novosibirsk $(\mathbf{N})$ and Sierra Leone $(\mathrm{S})$, by means of multiply marked chromosomes and back crossing. These parental strains are designated $\mathrm{Niav}^{+}, \mathrm{Siav}^{+}$, $\mathrm{Niav}^{-}$and Siav ${ }^{-}$, respectively.

To avoid the effects of inbreeding the $F_{1}$ individuals derived from a cross between appropriate wild type or iav parents are used, and for purposes of notation, the female parent is always designated first. Thus, a fly of the genotype $\mathrm{NS} \mathrm{iav}^{+}$is an $\mathrm{F}_{1}$ wild type individual derived from a Novosibirsk mother and Sierra Leone father. If no difference is found in the behaviour and/or survival of the reciprocal crosses (NS and $\mathrm{SN}$ ), then these scores are pooled for sex and genotype analyses.

The other strains used were not controlled for genetic background nor inbreeding. These are inactive ${ }^{2}$ $\left(\right.$ iav $^{2}$, formerly hypoactive- $\left.B^{1}\right)$, hypoactive-C (hypoC) and slow-receptor-potential ${ }^{4}\left(\right.$ slrp $^{4}$, formerly hypoactive- $D$ ) all isolated by Homyk \& Sheppard (1977) and exhibiting locomotor-deficient phenotypes; retinaldegeneration- $B^{23} \quad\left(r d g B^{23}\right.$ formerly hypoactive- $F$, Homyk et al., 1980); Oregon-R wild type; the selected low activity line La Mancha (van Dijken \& Scharloo, 1979 ); white ${ }^{1118}$ ( $w^{1118}$, Hazelrigg et al., 1984); shibire ${ }^{\text {ts }}$ (shits, Grigliatti et al., 1973); and two multiply marked lines constructed here in Glasgow, $w c t^{6}$ hypoC and iav $m f$, the latter containing the original iav allele of
Kaplan (1977). Further information on these mutations is contained in Lindsley \& Zimm (1992).

All flies were raised on standard oatmeal and molasses medium and maintained at $25 \pm 0.5^{\circ} \mathrm{C}$ on a 12-h light:dark cycle. The sexes were separated under ether (longevity and courtship) or $\mathrm{CO}_{2}$ (rhythm and $p$ Cresol survival) on the day of eclosion and maintained in groups of 10 in vials until required.

\section{Longevity}

Survival on standard medium was scored over a period of 15 weeks. Two hundred individuals of each sex (male and virgin female), genotype (wild type and inactive) and reciprocal cross (NS and $\mathrm{SN}$ ) were divided on eclosion into groups of 10 and retained in vials seeded with live yeast. This gives a total of 1600 individual records of survival. Survival at $25^{\circ} \mathrm{C}$ was scored every day and freshly yeasted medium was provided on every second day.

\section{Courtship of young males}

All courtships were observed in circular mating cells as described previously (O'Dell et al., 1989). The control (courting) flies were 3 days old $\mathrm{NS} \mathrm{iav}^{+}$wild type males. The target flies were single wild type $\left(\mathrm{SN} i a v^{+}\right)$ or $i a v\left(\mathrm{SN}_{i a v^{-}}\right)$males at 2, 26 or $50 \mathrm{~h}$ old. These ages were chosen so that courtship could be observed at the same time each day on flies that can essentially be described as 0,1 and 2 days old. Flies were kept singly in vials prior to their introduction to the mating chamber.

Preliminary analyses using forked mutant males (unpublished) confirm that courtship is exclusively expressed by the older ( 3 days old) male towards the younger male and that the reciprocal never occurs. Therefore, although the courting and target males are morphologically indistinguishable, we can be confident that the males expressing courtship are the 3-day-old $\mathrm{NS} \mathrm{iav}^{+}$wild type males.

Each morning, the behaviour of two control wild type courting males towards two target males, from each of the six classes, was observed. The order of the 12 trials was randomized and each trial was scored blind. The experiment was repeated on 10 mornings. The attractiveness of the target young males was measured using the sex appeal parameter (SAP) of Jallon \& Hotta (1979). The SAP is calculated as the time the courting (older) male spends directing wing vibration towards the target fly (in this case the immature male) during the first $5 \mathrm{~min}$ of courtship. Each fly was used only once. 


\section{Circadian rhythm}

The eclosion rhythm of two wild type $\left(\mathrm{NS} \mathrm{iav}^{+}\right.$and $\mathrm{SN} i a v^{+}$) and two iav (NSiav ${ }^{-}$and $\mathrm{SN} i a v^{-}$) strains was investigated using a protocol similar to that of Konopka \& Benzer (1971). The 12/12 h light:dark acclimatized parental generation were collected as virgins, paired in the light and allowed to lay in yeasted bottles for 3 days. The parents were then discarded and the cultures placed at $25^{\circ} \mathrm{C}$ in constant darkness. At midnight on day 9-10, all the bottles were emptied under red light. Eclosing flies were collected every $3 \mathrm{~h}$ for the following 4 days. A total of 4133 wild type flies and 5425 inactive mutants eclosed during the 96-h observation period.

\section{Effect of p-Cresol on survival}

The octopamine structural analogue $p$-Cresol (4methylphenol) was obtained from Sigma. As described earlier, dilutions of $p$-Cresol into the standard medium were made to obtain food with a $p$-Cresol concentration of 1 per cent by volume or less. Females aged 5-7 days were introduced to vials containing food with a variety of dilutions of $p$-Cresol and control vials containing food with no added $p$-Cresol. Ten flies were introduced to each vial, and five vials were observed for each genotype for each $p$-Cresol dilution. Survival at $25^{\circ} \mathrm{C}$ was recorded every hour.

\section{Results}

\section{Longevity}

The effect of the iav mutation on longevity is shown in Fig. 1. The lifespan of iav males and females is significantly shorter than that of the equivalent wild type flies $(F=171.38$, d.f. $=1, P<0.001)$. The iav mutation decreases the lifespan by approximately 6 days under these conditions.

Interestingly, males show a shorter lifespan than females, the effect being somewhat greater than that observed between the two genotypes $(F=371.13$, d.f. $=1, P<0.01)$. Under these conditions females outlive males by approximately 12 days. there is also a small but significant interaction between genotype and $\operatorname{sex}(F=5.52$, d.f. $=1, P<0.05)$, which is due to the greater difference in survival between wild type and iav females relative to males.

\section{Courtship of young males}

The results indicate that the attractiveness of wild type males decreases markedly with age, from an SAP of 0.223 , soon after eclosion, to 0.023 after $26 \mathrm{~h}$ and

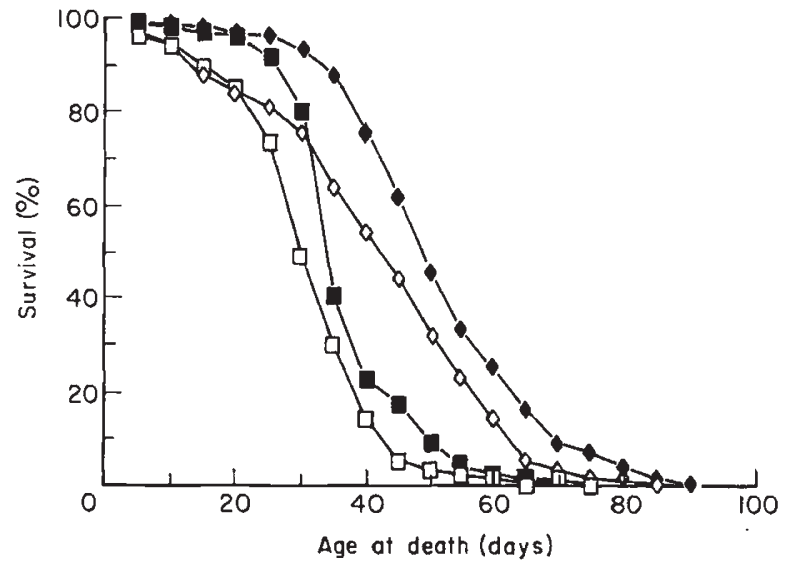

Fig. 1 Longevity of wild type and inactive males and females expressed as a percentage survival over age in days. Each point represents the data for 400 individuals. Mean longevities (and standard errors) in days are ( ) wild type male $35.60(0.50),(\diamond)$ wild type female $49.98(0.72),(\square)$ iav male $30.04(0.52),(\diamond)$ iav female $41.28(0.87)$.

0.002 after $50 \mathrm{~h}$. The attractiveness of iav males also decreases with age over the same period from an SAP of 0.351 at $2 \mathrm{~h}$, to 0.115 at $26 \mathrm{~h}$ and 0.053 at $50 \mathrm{~h}$.

However, for each age examined the iav male is more attractive than its equivalent wild type male, the difference being most noticeable in the older $(50 \mathrm{~h})$ males. Here the iav males elicit about 25 times more wing vibration than their wild type counterpart. In fact the $50 \mathrm{~h}$ iav male elicits twice as much courtship as a $26 \mathrm{~h}$ wild type male.

An analysis of variance confirms the results alluded to above. As expected, attractiveness decreases with age $(F=92.11$, d.f. $=2, P<0.01)$ and wild type males are significantly less attractive than iav males $(F=29.12$, d.f. $=1, P<0.01)$. The observation that there is no significant interaction between age and genotype $(F=1.76$, d.f. $=2, p=n s)$, confirms there is a similar pattern of decreasing attractiveness with age for both wild type and iav males.

\section{Circadian rhythm}

The eclosion rhythms of wild type and iav mutant flies are presented in Fig. 2. As no differences were found between the rhythms of males and females nor between the genetic backgrounds of the parents, data were pooled for the two sexes and reciprocal crosses.

The results indicate that wild type flies show a 24-h eclosion rhythm. The peak 3-h eclosion rate is always between the hours of 09.00 and 12.00 , immediately after perceived 'lights on'. The low point of eclosion is between 24.00 hours and 03.00 , the middle of perceived 'night-time'. 

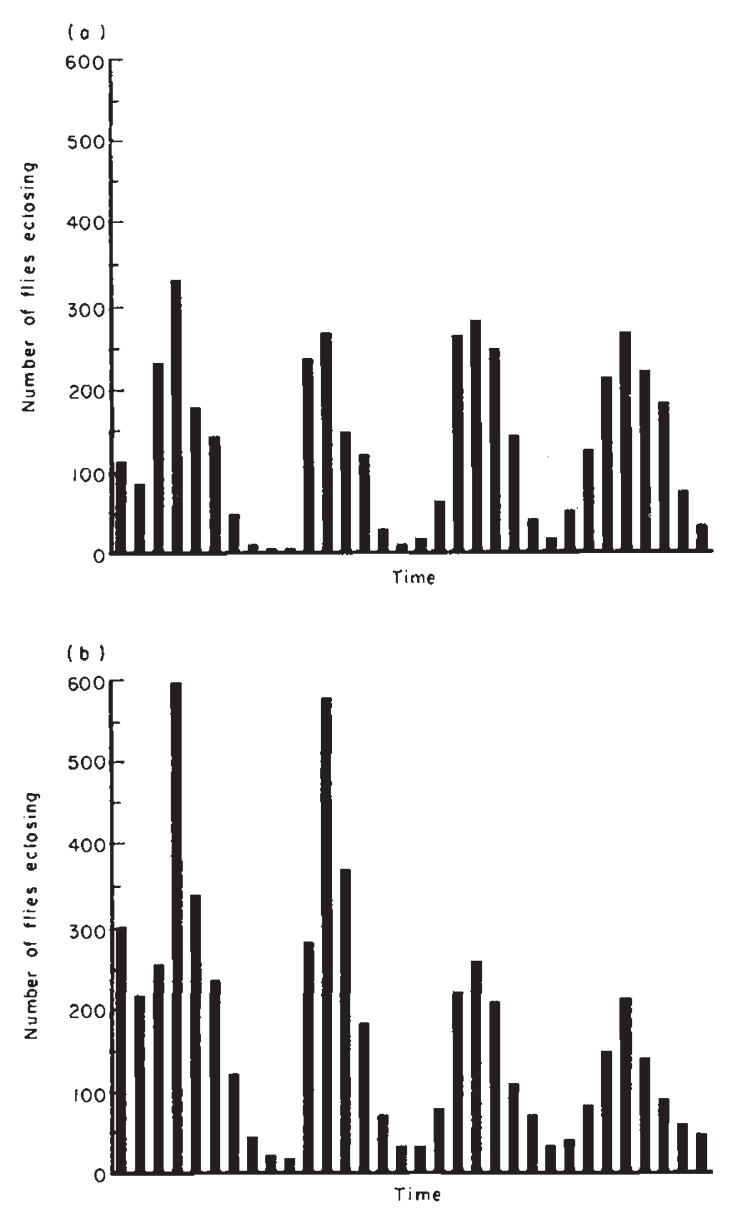

Fig. 2 Eclosion rhythm of (a) wild type and (b) inactive flies. The bars represent the number of flies eclosing in each 3-h period starting at midnight on day 1 and continuing for 4 days.

The pattern of eclosion of iav mutants is similar to that of the wild type flies. Indeed the peaks and troughs of iav eclosion occur during precisely the same 3-h periods as wild type eclosion on each of the 4 days of observation. Clearly, iav mutants have a normal eclosion rhythm.

\section{Effect of p-Cresol on survival}

Preliminary trials showed that food medium supplemented with $p$-Cresol has a deleterious affect on the survival of both wild type and iav mutant females, as vials containing no $p$-Cresol show little or no mortality over the same observation period (data not shown). As revealed by Student's $t$-test, the survival rate of iav females is markedly reduced with respect to wild type females $(t=8.30$, d.f. $=49, P<0.001)$. Indeed, the mean length of survival of iav females on 0.5 per cent $p$-Cresol is only $1.62 \mathrm{~h}$ compared to $4.46 \mathrm{~h}$ of wild type females.

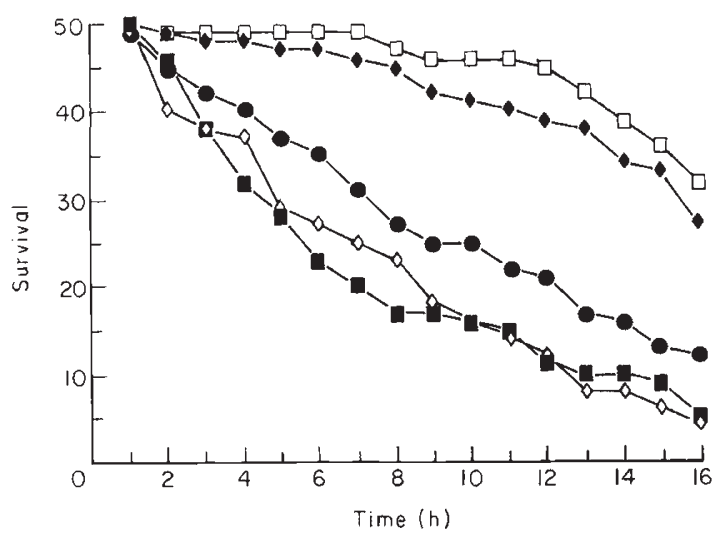

Fig. 3 Survival of $(\square)$ wild type, ( $) i a v^{1},(\diamond) i a v^{2}$, females and their hybrids $\left(\bullet, i a v^{+} / i a v^{\prime} ; \bullet, i a v^{1} / i a v^{2}\right)$ on medium containing $0.5 \%$-Cresol. $n=50$.

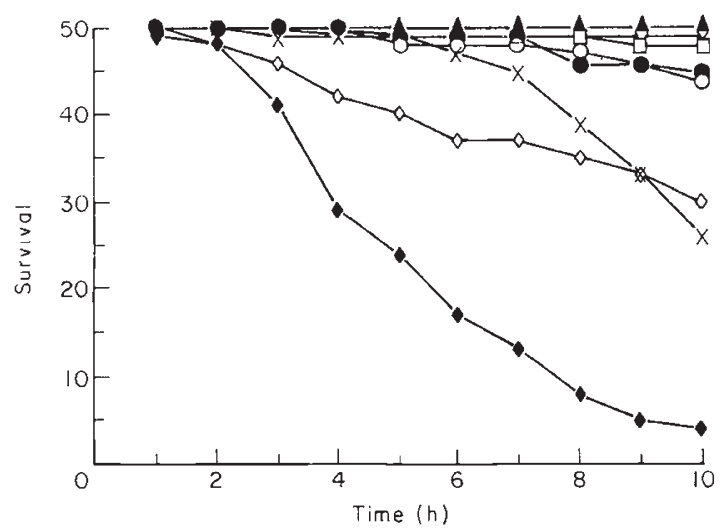

Fig. 4 Survival of females on medium containing $0.5 \% p$ -

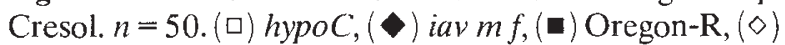
$r d g B^{23},(\bigcirc) s h i^{\mathrm{ts}},(\bullet) \operatorname{slr}^{4},(\mathbf{\Delta})$ La Mancha, $(\Delta) w c t^{6}$ hypoC, $(\times) w^{1118}$.

Females carrying the $i a v^{2}$ allele show a susceptibility to $p$-Cresol that is indistinguishable from that of iav females (Fig. 3). Exposure to $p$-Cresol is similarly deleterious to the survival of $F_{1}$ hybrid $i a v / i a v^{2}$ females. $\mathrm{F}_{1}$ hybrid $\mathrm{iav}^{+} /$iav females show wild type like resistance to $p$-Cresol.

The data in Fig. 4 confirm that the deleterious effect of $p$-Cresol is specific to flies carrying a mutation in the iav gene. It shows that flies with a locomotor-deficient phenotype, superficially similar to that of iav, are indistinguishable from wild type flies in their response to $p$ Cresol. This is irrespective of their relative inactivity being associated with a single mutation (hypoC, $r d g B^{23}$, $s l r p^{4}$ and $\left.w^{1118}\right)$, several mutations $\left(w c t^{6}\right.$ hypoC) or resulting from an extended period of selection (La Mancha). The multiply marked strain $(\operatorname{iav} m \mathrm{f})$, derived from the original iav mutation, is similar in its susceptibility to $p$-Cresol to the isogenized iav line. 


\section{Discussion}

\section{Longevity}

It is a prediction of the 'Rate of Living' hypothesis of Pearl (1928) that activity should be inversely related to age at death. Trout \& Kaplan (1970) demonstrated just such an effect in the relatively short-lived but hyperactive Drosophila mutant Shaker. The problem in measuring the expression of longevity in mutant lines of $D$. melanogaster is the marked sensitivity of the phenotype to the external environment, particularly crowding, and effects of genetic background (Lamb, 1978). However, in these trials these problems have largely been eliminated.

The observation that wild type flies have a longer life span than the hypoactive iav mutants clearly runs contrary to the 'Rate of Living' hypothesis. However, the extended longevities of females relative to males fits the observations of several authors (Allemand et al., 1973; Maynard Smith, 1958). Why this should be so is unclear.

Although the 'Rate of Living' hypothesis has been the subject of some recent criticism (Le Bourg, 1987), the observation that phenotypically inactive flies have a shorter lifespan than wild type individuals need not necessarily be taken as evidence against the hypothesis. The apparent reduction in iav mutant longevity may be a consequence of an, as yet, unknown function of the iav gene. Given that the genotypic difference in longevity is greater between wild type and iav females than between males, it may also indicate some femalespecific function of the iav gene. Alternatively, the small decrease in iav mutant longevity may reflect some aspect of their inactive behavioural phenotype that contributes to an age-independent increase in their mortality.

\section{Courtship of young males}

Observations on the effect of ageing on locomotor activity and female sexual receptivity suggest there is a short delay in the onset of mature adult behaviour in young iav adults (O'Dell \& Burnet, 1988; O'Dell et al., 1989). The delay in the onset of female receptivity may be associated with the late occurrence of 'switch on', that is the point at which young virgin females change from an unreceptive to receptive state (Manning, 1967). However, no delay in maturation has yet been demonstrated in any aspect of the courtship behaviour of iav males.

Detailed analyses of Drosophila courtship have revealed the unexplained phenomenon of male/male courtship. Males that are only a few hours old stimulate older males to court them (Cook \& Cook, 1975). This occurs in all D. melanogaster strains so far investigated (McRobert \& Tompkins, 1983) and is due to the production of female-like sex pheromones by the young males (Tompkins et al., 1980; Jallon, 1984). However, this attractiveness falls rapidly and 2-day-old wild type males elicit little or no courtship from older males (Jallon \& Hotta, 1979).

If the iav mutation has a consequence for some or all aspects of maturation in newly eclosed adults, then we might expect young iav males to exhibit extended attractiveness. This appears to be the case as iav males are still highly attractive 2 days after eclosion, whereas wild type males are not.

These results show that iav males are more attractive than wild type males at all three ages investigated. This may be due in part to their inactive phenotype, which largely precludes their escaping the attention of older courting males. Similar observations have been made in courtships involving virgin iav females, which attract a high frequency of wing vibration from courting males. This is thought to be a consequence of their relative inactivity, which precludes their decamping and other avoidance and escape behaviours (O'Dell $e t$ al., 1989). However, the observation that the relative attractiveness of iav males to wild type increases over time, clearly demonstrates extended attractiveness.

Males carrying the fruitless (fru, Tompkins et al., 1980; Gailey \& Hall, 1989) or apterous (ap, Jallon \& Hotta, 1979) mutations are attractive to mature males throughout their lives. In both cases this is associated with the permanent presence of the immature male pheromone coat. In fact solvent cuticular extracts of old fru males are sufficient to stimulate other mature males to display courtship behaviour (Tompkins et al., 1980). The presence of extended attractiveness in young iav males suggests there may be some delay in the switch of the iav male pheromonal coat from the immature to mature state. The pheromone coat of 4-day-old iav males is indistinguishable from that of wild type (O'Dell et al., 1989) but the nature of the pheromone coat of the attractive 2-day-old iav males used here is unknown. However, the observation that young iav males exhibit extended attractiveness supports the hypothesis that the iav mutation delays post-eclosional maturation.

\section{Circadian rhythm}

According to Livingstone \& Tempel (1983), the circadian rhythm mutant period (Konopka \& Benzer, 1971) has a small deficit in octopamine. If octopamine is the cause rather than a consequence of the aberrant circadian rhythm then one might reasonably expect iav mutants, which have only 15 per cent wild type octopa- 
mine (O'Dell et al., 1987), to exhibit a rhythmless phenotype. However, wild type and iav mutant eclosion rhythms are indistinguishable, and given that iav flies also show a normal locomotor rhythm (B. Kyriacou, personal communication), it is unlikely that octopamine has any causative role in the expression or maintenance of normal circadian rhythm. The small deficit in octopamine in per mutants is therefore unlikely to have a causative role in the expression of their aberrant rhythm phenotype.

\section{Effect of $p$-Cresol on survival}

The observation that iav mutants have only 15 per cent of the wild type levels of octopamine but normal dopamine titres (O'Dell et al., 1987), suggests that the iav gene product may be involved in the synthesis of octopamine from tyrosine, presumably via tyramine (see review by Wright, 1987). A candidate gene product for the iav gene would therefore be tyramine $\beta$ hydroxylase (TBH), the enzyme that converts tyramine into octopamine.

In invertebrates, TBH is thought to play a role analogous to that of dopamine $\beta$-hydroxylase $(\mathrm{DBH})$ in mammals (see Fig. 5). Indeed the TBH and $\mathrm{DBH}$ enzymes are thought to share evolutionary history, an observation that is supported by the characterization of lobster TBH by Wallace (1976). As a consequence of this similarity in DBH and TBH function and ancestry, it may be possible to use inhibitors of vertebrate $\mathrm{DBH}$ to investigate the alleged role of TBH in iav mutants.

Work on mechanism-based inactivation of the bovine DBH enzyme has revealed two inhibitors of enzyme activity, $p$-Cresol (Goodhart et al., 1987) and
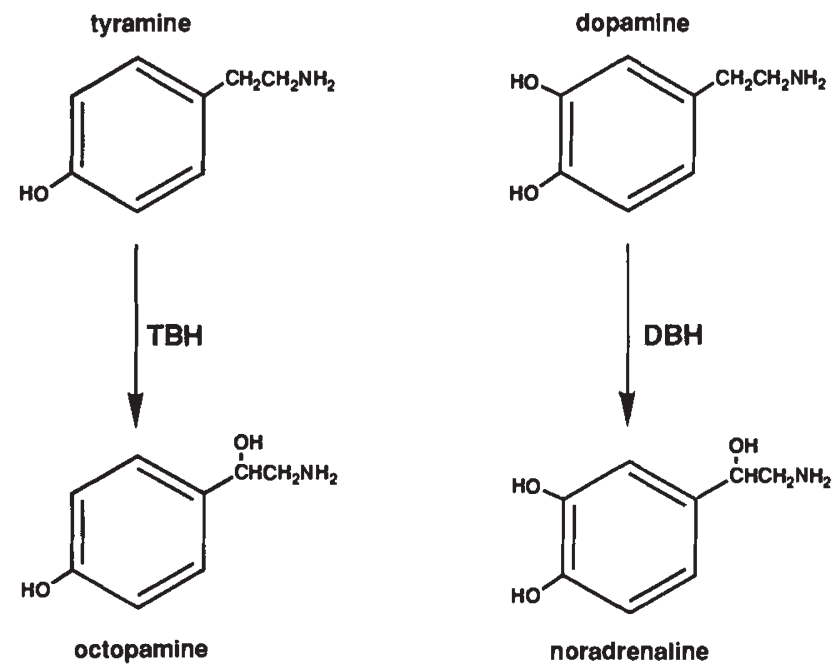

Fig. 5 Reactions catalysed by dopamine $\beta$-hydroxylase $(\mathrm{DBH})$ and tyramine $\beta$-hydroxylase $(\mathrm{TBH})$. $\beta$-ethynyltyramine (DeWolf et al., 1989). $p$-Cresol is a simple structural analogue of dopamine which lacks a basic side chain nitrogen and binds DBH in a mechanism that is indistinguishable from normal dopamine substrate binding (Goodhart et al., 1987). If $p$-Cresol binds to and inactivates Drosophila TBH in a manner analogous to that for bovine DBH, then flies deficient for TBH may find it more toxic than do wild type flies.

All the lines that show susceptibility to $p$-Cresol have a mutation in the iav gene (iav, iav $v^{2}$ and iav $m f$ ). Heterozygous $i a v^{+} / i a v$ females show wild type levels of resistance to $p$-Cresol, confirming the recessive nature of the iav allele. Given that other phenotypically inactive lines, such as La Mancha and $w^{1118}$, show wild type survival rates, the mode of action of $p$-Cresol must be a consequence of mutations in the iav gene rather than a consequence of their inactive phenotype.

These data support both the notion that an octopamine deficit is associated with the iav phenotype and the possibility that $\mathrm{TBH}$ is the iav gene product. Alternatively, $p$-Cresol may mediate its effect by mimicking and out-competing the action of octopamine. In iav mutants, the relative scarcity of octopamine would allow $p$-Cresol to mediate its toxic effect at lower concentrations. It therefore seems likely that the behavioural abnormalities associated with the iav mutation are a consequence, rather than a cause of a deficit in octopamine.

\section{Acknowledgements}

This work was made possible by the award of a Wolfson Special Grant to David Sherratt FRS. The author would also like to thank Barrie Burnet, David Roberts, Bambos Kyriacou, Jane Davies and Marshall Stark whose help and advice is gratefully acknowledged.

\section{References}

ALLEMAND, R., COHET, Y. AND DAVID, J. R. 1973. Increase in the longevity of adult Drosophila melanogaster kept in permanent darkness. Exp. Geront., 8, 279-283.

COOK, R. M. AND COOK, A. 1975. The attractiveness to males of female Drosophila melanogaster: effects of mating, age and diet. Anim. Behav., 23, 521-526.

DAVID, J. C. AND COULON, J. F. 1985. Octopamine in invertebrates and vertebrates. Prog. Neurobiol., 24, 141-185.

DeWOLF, W. E., CHAMBERS, P. A., SOUTHRAN, C., SAUNDERS, D. AND KRUSE, L. I. 1989. Inactivation of dopamine $\beta$-hydroxylase by $\beta$-ethynyltyramine: kinetic characterization and covalent modification of active site peptides. Biochemistry, 28, 3833-3842.

GAILEY, D. A. AND HALL, J. C. 1989. Behavior and cytogenetics of fruitless in Drosophila melanogaster: different courtship 
defects caused by separate, closely linked lesions. Genetics, 121, 773-785.

GOODHART, P. J., DeWOLF, w. E. AND KRUSE, L. I. 1987. Mechanismbased inactivation of dopamine $\beta$-hydroxylase by $p$ Cresol and related alkylphenols. Biochemistry, 26, 2576-2583.

GRIGLIATTI, T. A., HALL, L. M. C., ROSENBLUTH, R. AND SUZUKI, D. T. 1973. Temperature-sensitive mutations in Drosophila melanogaster. XIV. A selection of immobile adults. $\mathrm{Mol}$. Gen. Genet., 120, 107-114.

HALL, J. C. 1982. Genetics of the nervous system in Drosophila. Q. Rev. Biophys., 15, 223-479.

HAZELRIGG, T. 1., LEWIS, R. AND RUBIN, G. M. 1984. Transformation of white locus DNA in Drosophila: dosage compensation, zeste interaction and position effects. Cell, 36, 469-481.

номүк, т. 1977. Behavioral mutants of Drosophila melanogaster. II. Behavioral analysis and focus mapping. Genetics, 87, 105-128.

HOMYK, T. AND SHEPPARD, D. E. 1977. Behavioral mutants of Drosophila melanogaster. I. Isolation and mapping of mutations that decrease flight ability. Genetics, 87, 95-104.

HOMYK, T., SZIDONYA, J. AND SUZUKI, D. T. 1980. Behavioral mutants of Drosophila melanogaster. III. Isolation and mapping of mutants by direct visual observations of behavioral phenotype. Mol. Gen. Genet., 177, 553-565.

JALLON, J.-M. 1984. A few chemical words exchanged by Drosophila during courtship and mating. Behav. Genet, 14, 441-478.

JALLON, J.-M. AND HOTTA, Y. 1979. Genetic and behavioral studies of female sex appeal in Drosophila. Behav., Genet., 9, 257-275.

KAPLAN, w. D. 1977. iav: inactive. Drosophila Inf. Serv., 52, 1.

KONOPKA, R. J. AND BENZER, s. 1971. Clock mutants of Drosophila melanogaster. Proc. Natl. Acad. Sci. U.S.A., 68, 2112-2116.

LAMB, M. J. 1978. Ageing. In: Ashburner, M. and Wright, T. H. F. (eds) Genetics and Biology of Drosophila, Vol. 2c, Academic Press, New York, pp. 43-104.

LE BOURG, E. 1987. The rate of living theory, spontaneous locomotor activity, ageing and longevity in Drosophila melanogaster. Exp. Gerontol., 22, 359-369.
LINDSLEY, D. AND Zimm, G. 1992. The Genome of Drosophila melanogaster. Academic Press, CA.

LIVINGSTONE, M. S. AND TEMPEL, B. L. 1983. Genetic dissection of monoamine neurotransmitter synthesis in Drosophila. Nature, 303, 67-70.

McROBERT, S. P. AND TOMPKINS, L. 1983. Courtship of young males is ubiquitous in Drosophila melanogaster. Behav. Genet., 13, 517-523.

MANNING, A. 1967. The control of female receptivity in Drosophila. Anim. Behav., 15, 239-250.

MAYNARD SMITH, J. 1958. The effects of temperature and of egg-laying on the longevity of Drosophila subobscura. $J$. Exp. Biol., 35, 832-842.

O'DELL, K. M. C. AND BURNET, B. 1988. The effects on locomotor activity and reactivity of the hypoactive and inactive mutations of Drosophila melanogaster. Heredity, 61, 199-207.

O'DELL, K. M. C., COULON, J.-F., DAVID, J.-C., PAPIN, C., FUZEAUBRAESCH, S. AND JALLON, J.-M. 1987. La mutation inactive produit une diminution marquee d'octopamine dans le cerveau des Drosophiles. C.R. Acad. Sci. Paris, Ser. III, 305, 199-202.

O'DELL, K. M. C., BURNET, B. AND JALLON, J.-M. 1989. Effects of the hypoactive and inactive mutations on mating success in Drosophila melanogaster. Heredity, 62, 373-381.

PEARL, R. 1928. The Rate of Living. University of London Press, London.

TOMPKINS, L., HALL, J. C. AND HALL, L. M. C. 1980. Courtshipstimulating volatile compounds from normal and mutant Drosophila. J. Insect. Physiol., 26, 689-697.

TROUT, w. E. AND KAPLAN, w. D. 1970. A relationship between longevity, metabolic rate and activity in Shaker mutants of Drosophila melanogaster. Exp. Gerontol., 5, 83-92.

VAN DIJKEN, F. R. AND SCHARLOO, w. 1979. Divergent selection on locomotor activity in Drosophila melanogaster. I. Selection response. Behav. Genet., 9, 543-553.

WALLACE, B. G. 1976. The biosynthesis of octopamine: characterization of lobster tyramine $\beta$-hydroxylase. $J$. Neurochem., 26, 761-770.

WRIGHT, T. H. F. 1987. The genetics of biogenic amine metabolism, sclerotization and melanization in Drosophila melanogaster. In: Scandalios, J. G. (ed.) Advances in Genetics, Vol. 24, Academic Press, CA. 